Voix et Images

\title{
La Polémique autour de la Voix d'un exilé ou le Chant du cygne de l’immanentisme au Québec
}

\section{Guy Monette}

Volume 2, numéro 3, avril 1977

Jean Éthier-Blais

URI : https://id.erudit.org/iderudit/200068ar

DOI : https://doi.org/10.7202/200068ar

Aller au sommaire du numéro

Éditeur(s)

Les Presses de l'Université du Québec

ISSN

0318-9201 (imprimé)

1705-933X (numérique)

Découvrir la revue

Citer cet article

Monette, G. (1977). La Polémique autour de la Voix d'un exilé ou le Chant du cygne de l'immanentisme au Québec. Voix et Images, 2(3), 334-357.

https://doi.org/10.7202/200068ar d'utilisation que vous pouvez consulter en ligne.

https://apropos.erudit.org/fr/usagers/politique-dutilisation/ 


\section{La Polémique autour de la Voix d'un exilé ou le Chant du cygne de l'immanentisme au Québec}

à Alphee Bake et Jean-Paul Hudon

Le destin s'acharne parfois sur certaines œuvres littéraires. Généralement, un tel sort ne découle pas uniquement du caractère de l'œuvre ou de la personnalité de l'auteur, mais tient très souvent à la conjoncture historique dans laquelle l'œuvre et l'auteur s'insèrent. La Voix d'un exilé du poète Louis-Honoré Fréchette nous est apparue comme une de ces œuvres qui, sans être particulièrement remarquables quant à leur style, jouissent cependant d'un statut spécial dans le monde des lettres à cause de leur charge signifiante et de leur fortune littéraire.

Personne ne saurait nier que la décennie des années soixante du XIXe siècle soit une période cruciale de l'histoire du Québec. Or la Voix d'un exilé s'inscrit dans cette période d'effervescence généralisée qu'alimentent deux grands courants de pensée. En effet, que ce soit au sujet des luttes électorales, du projet et de la mise sur pied de la Confédération, des conceptions religieuses, littéraires ou philosophiques, de l'urbanisation et de l'industrialisation naissantes, du droit à la libre lecture, de l'émigration des Québécois, des rebondissements de la vie publique européenne, des institutions sociales ou encore de l'enterrement de l'imprimeur Guibord, des luttes surgissent où s'affrontent deux positions constamment contradictoires. Lors de chacun des événements dont foisonnent ces quelques années, deux groupes d'adversaires se dressent l'un contre.l'autre et tentent d'amener à leurs vues une population qui assiste, médusée, à des combats qui scelleront pour longtemps son avenir.

Les recherches effectuées sur cette période ont rendu familière la toponymie des divers aspects de ces luttes. Bien que fort valables, les doublets du type conservatisme/libéralisme, conservatisme clérical/rougisme, ultramontanisme/libéralisme religieux, élitisme/démocratie, autoritarisme/libertarisme, classicisme/romantisme, confédéralisme/annexionnisme ou indépendantisme, ont cependant le tort d'être trop restrictifs. L'ensemble des diverses tendances que recouvrent ces dénominations peut effectivement se regrouper sous deux grandes catégories qui ont le mérite d'effacer des divergences factuelles pour laisser apparaître une nature commune à des phénomènes de prime abord fort éloignés. Tous les premiers 
éléments de la série d'antonymes mentionnée plus haut recourent en effet à des arguments fondés sur une autorité reconnue et reposent sur une intemporalité, alors que les deuxièmes se réclament plutôt du réel immédiat. Or, une conception quelconque qui invoque Dieu, des valeurs historiques ou l'autorité des pouvoirs établis et s'appuie sur.eux pour se justifier, peut être décrite comme "transcendantiste", tandis que sa contrepartie, qui tente de se fonder dans une analyse situationnelle, peut être qualifiée, quant à elle, d' «immanentiste ${ }^{1}$ ".

Durant ces années soixante, nous assisterions à la dernière confrontation importante entre un immanentisme récent et un transcendantisme solidement implanté qui se refuse à toute contestation. De nombreux événements de cette époque semblent propres à étayer cette hypothèse; mais tous, à l'exception d'un seul, présentaient le défaut de laisser au second plan les textes suscités par l'une ou l'autre des composantes essentielles de cette confrontation. Ce fait unique, Louis-Honoré Fréchette nous l'offre lorsqu'il publie la Voix d'un exilé. Une réflexion sur ce texte et sur les textes suscités par la polémique qui mit aux prises l'auteur et Adolphe-Basile Routhier, nous permet aujourd'hui de mieux comprendre à quel point cette période constitue une véritable charnièrè dans l'histoire des idées au Québec.

Par sa situation historique et la notoriété de son auteur, la Voix d'un exilé avait déjà mérité un examen critique; mais le contenu de l'œuvre et sa signification ont fait peser sur elle une fatalité exceptionnelle dans notre univers littéraire. En effet, la Voix fut presque totalement scotomisée dans l'histoire de notre littérature. Une idéologie triomphante aurait voulu effacer jusqu'au dernier sursaut littéraire significatif d'une rivale vaincue, qu'elle n'aurait pas procédé autrement. Mais aujourd'hui, en cette ère de redécouverte du $X I X^{e}$ siècle québécois, il est grand temps d'exhumer ce texte négligé par l'histoire littéraire et qui mérite au moins une relecture; sinon une republication.

Un tour d'horizon de la critique nous permettra de mieux comprendre la portée d'un silence lourd de signification et de conséquences ainsi que d'illustrer l'étrange destin de cette œuvre dont Henri d'Arles a pu écrire: “C'est elle, c'est cette petite chose mystérieuse, introuvable, devenue curiosité bibliographique, que presque personne n'a vue, ni lue, que l'on ne connaît que par de vagues échos, c'est cela qui a entouré le nom de Fréchette de gloire ${ }^{2}$." La gloire de Fréchette est peut-être demeurée mais l'œuvre a été oubliée. En effet, cet “opuscule rarissime ${ }^{3}$ ", selon l'expression d'Auguste Viatte, reçoit tout au plus une simple mention dans les manuels d'histoire littéraire. Les quelques auteurs qui s'y attardent, se contentent généralement de souligner sa ressemblance avec les Chatiments de Victor Hugo; mais, bien qu'ils en apprécient parfois le style fougueux, ils condamnent unanimement le contenu du poème ${ }^{4}$.

La rareté du texte de la Voix n'explique que partiellement cette méconnaissance trop généralisée pour être entièrement involontaire. 
Lors de la première publication en 1866, «Fréchette édita son livre luimême avec une presse hydraulique ${ }^{\text {" }}$ et, il va sans dire que, parue à Chicago et ne comportant que huit pages, cette édition n'existe probablement plus. Les deuxième et troisième éditions, comportant respectivement dix-huit et quarante-six pages, furent également publiées à Chicago à l'imprimerie du journal l'Amérique en 1868 et 1869 . Une quatrième édition de la Voix parut à Montréal en 1877 dans le recueil de Fréchette intitulé Pèle-mêle et publié chez John Lowell; toutefois, sur l'ensemble de ce tirage, une centaine de volumes seulement inclut la Voix d'un exilé à la fin du livre sous la rubrique "poésies politiques». Les éditions de 1868 et 1877 comportent en outre un poème intitulé le Premier Coup de foudre qui ne fait pas directement partie de la Voix d'un exilé.

Certains volumes de référence mentionnent une édition de cinquantesix pages qui aurait été faite à Chicago en 1868 sans la connaissance de l'auteur ${ }^{6}$. La chose paraît peu probable, d'une part parce que l'on ne retrouve pas trace d'une telle édition et d'autre part parce que Routhier affirme, dans les Causeries du dimanche ${ }^{7}$, avoir reçu de Fréchette lui-même les éditions de 1868 et 1869 . D'ailleurs, l'édition de 1869 dont l'exemplaire que nous avons consulté comporte une dédicace manuscrite de Fréchette à Longfellow et qui ne couvre que quarante-six pages, date l'achèvement du poème d'avril 1869. II aurait alors été difficile de publier cinquante-six pages de texte en 1868. Un doute pourrait cependant subsister puisque, dans l'édition de 1877 , Fréchette date les trois parties de son poème respectivement de 1866, 1867 et 1868 ; il est probable qu'il s'agisse tout simplement là d'une erreur volontaire de l'auteur qui aurait ainsi voulu souligner la régularité de sa production. De toute façon, les éditions de 1868 et 1869 datent la deuxième partie du poème du 8 avril 1868 ou de mai 1868, ce qui invalide la chronologie de l'édition de 1877 et confirme l'impossibilité de l'existence d'une édition pirate de cinquante-six pages en 1868.

On s'étonne donc qu'une œuvre qui reçut quatre éditions ${ }^{B}$ et fut reproduite dans les journaux canadiens ${ }^{9}$ et même européens ${ }^{10}$, puisse être introuvable. Certes le temps n'a pas ménagé les volumes du XIX ${ }^{\mathrm{e}}$ siècle québécois; quant aux journaux, ils sont de nature éphémère. Cependant un tel sort ne saurait être le lot d'une seule œuvre. Le caractère polémique de la Voix d'un exilé laisse sans doute soupçonner des destructions dues à des adversaires; mais que Fréchette lui-même se soit fait l'instrument du destin est plus surprenant. Le poète "rachetait même à prix d'or " les exemplaires de la Voix d'un exilé qu'«il pouvait dénicher et les jetait au feu 11 ". Fréchette refusa même à Augustin Laperrière la permission de reproduire le texte dans les Guêpes canadiennes ${ }^{12}$.

En dépit de ces avanies, quelques critiques ont cependant cru que ce texte méritait plus d'attention. Toutefois leurs diverses analyses, souvent contradictoires, révèlent le climat de méfiance qui entourait jusqu'à très récemment cette œuvre. Penchons-nous sur cette critique et voyons comment elle confirme les appréhensions des historiens de la littérature. 
La première critique étoffée, celle d'Adolphe-Basile Routhier, prend le contre-pied de l'œuvre et fera l'objet d'une partie de notre étude. Viendront ensuite en 1874 les commentaires d'Edmond Lareau qui croit que "les précieuses qualités de $M$. Fréchette se sont manifestées d'une manière éclatante dans la Voix d'un Exilé " et "qu'il serait difficile, à tout critique impartial, de juger Fréchette autrement et [le critique] en appelle à tous ceux qui ne veulent pas voir à l'aide des lunettes de notre politique ${ }^{13}$ ”. En 1894, le poète William Chapman éreintera pour sa part la Voix, ainsi que toute la production de Fréchette, sous le poids d'une accusation de plagiat. Fréchette aurait mis dans la Voix "une bonne moitié des Chatiments de Hugo" et serait comme «le molosse qui, l'écume de la rage à la gueule, cherche à mordre le passant ${ }^{14} \%$. Henri d'Arles, vers 1900 , tout en considérant la Voix comme «une monstrueuse erreur de jugement " où les «déboires personnels déforment la vision à un degré inouï» admet qu'on y trouve «du souffle, un lyrisme échevelé mais réel». (Arles, p. 11-12) Quant à Camille Roy, il voit dans le poème de Fréchette des «accents oratoires", une «ardente passion", des «tirades vengeresses, furieuses, échevelées", un «lyrisme tapageur" ainsi qu'une «imitation trop puérile des Chatiments ; en outre, Roy dénonce vertement “l'engouement pour la révolution" dont fait preuve l'auteur qui a cédé à "la mode du libéralisme et de l'incrédulité ${ }^{15}$ ". Marcel Dugas, bien qu'il considère que la Voix n'a aucune valeur littéraire, s'y intéresse cependant dans son étude publiée en 1946 et fournit des vues pertinentes sur la polémique entre Fréchette et Routhier. En 1958, Séraphin Marion éprouve toujours quelque malaise face à la thématique de l'œuvre et approuve le jugement qu'en a formulé Routhier presqu'un siècle plus tôt: "Rien - ou presque rien dans cette analyse de Routhier qui ne soit dans la ligne du bon sens." (Marion, p. 82) Marion reconnaît tout de même qu' "on peut néanmoins se demander si ce n'est pas encore dans ces strophes vitrioliques que Fréchette s'apparente le moins mal à Victor Hugo". (Marion, p. 26) Dans son Fréchette de 1959, Michel Dassonville se contente de reproduire dixneuf strophes de l'édition de 1868 de la Voix, accompagnées de variantes de l'édition de 1877. Cet auteur affirme que l'édition de 1868 constitue l'édition originale de la Voix et il ignore celle de $1869^{16}$. A l'encontre de Dassonville, il faut préciser que, si l'édition originale de 1866 est introuvable, elle est cependant reproduite dans le journal le Pays du 27 mars 1867. Cette reproduction, à défaut de mieux, peut être considérée comme l'édition originale de la Voix d'un exilé. Les commentaires les plus récents sur la Voix, ceux de Gérard Bessette, s'apparentent fort à ceux de Lareau; Bessette déclare en effet que "ce recueil constitue un des meilleurs textes de Fréchette, grâce à l'abondance des images, à son éloquence enflammée, à la richesse du vocabulaire ${ }^{17}$ ". Après ce regard sur la critique, il nous faut donc souscrire à l'opinion que Georges A. Klinck formulait en 1955 dans son étude sur la prose de.Fréchette; cet auteur écrit à propos des Lettres à Basile qu'elles “ont beaucoup plus qu'un intérêt académique. Elles soulèvent bien des questions qui nous embarrassent même en ce siècle de lumières $[\ldots]^{18} "$. Cette phrase s'applique également à la Voix 
d'un exilé et décrit avec justesse l'attitude ambiguë de la critique envers elle.

Silence des historiens de la littérature d'une part, malaise et contradiction chez la critique d'autre part: qu'un texte d'un poète qui fut considéré comme le barde national du Québec soit aussi diversement traité a de quoi piquer la curiosité et éveiller l'intérêt. Cette situation ne révéleraitelle pas le souvenir d'un conflit majeur dont les ramifications seraient encore aujourd'hui perceptibles? Une simple présentation du texte de la Voix lèvera peut-être ces ambiguittés et nous permettra de redécouvrir un texte probablement capital à la bonne compréhension du XIX siècle québécois. II est dommage, par exemple, que l'étude de Joseph Costisella sur l'esprit révolutionnaire au $X \mid X^{\theta}$ siècle dans laquelle l'auteur considère que Fréchette est le grand représentant littéraire de cette pensée, ne mentionne même pas la Voix qui est sans contredit l'œuvre la plus radicale de Fréchette ${ }^{19}$. II est donc plus qu'impérieux de restituer à la Voix la place qui lui revient dans notre histoire littéraire.

Mais qu'est-ce donc que cette cuvre et pourquoi a-t-elle engendré une polémique violente entre ces deux amis que furent Routhier et Fréchette? Qu'est-ce donc qui a pu ainsi secouer les lettres québécoises naissantes et dont le choc s'est perpétué jusqu'à nos jours? Pourquoi Routhier se sentit-il tenu de critiquer durement cette œuvre dans le Courrier du Canada et ensuite d'inclure ces articles dans son volume publié en 1871 et intitulé les Causeries du dimanche? Pourquoi Fréchette crut-il bon à son tour d'attaquer les Causeries du dimanche dans huit lettres publiées par l'Événement du 13 novembre 1871 au 16 janvier 1872 et de réunir ces lettres en volume sous le titre Lettres à Basile 20 ? Pourquoi Routhier réplique-t-il treize fois aux lettres de Fréchette dans le Nouveau Monde du 23 novembre 1871 au 12 février 1872²1 ? Qu'est-ce qui pousse LouisAntoine Dessaules ${ }^{22}$ et Arthur Buies ${ }^{23}$ à prendre part au combat? Quel intérêt les journaux de l'époque avaient-ils à faire du battage autour des deux jouteurs? Pourquoi Routhier renouvelle-t-il ses attaques contre Fréchette en $1873^{24}$ ? II importe de faire la lumière sur toutes ces questions qui nous permettrons de mieux situer la Voix et d'en comprendre la portée.

Louis-Honoré Fréchette est suffisamment connu pour ne pas avoir à être présenté longuement. Né en 1839, le jeune Fréchette poursuit des études quelque peu mouvementées pour enfin terminer son droit en 1864. Le futur avocat s'intéresse autant sinon plus à la littérature qu'au droit. II fréquente le cénacle littéraire qu'était le groupe patriotique de Québec. Partois en compagnie de son condisciple et ami Routhier, il s'initie par la lecture de Lamartine et Hugo au romantisme français. Jeune avocat sans cause, il s'occupe de journalisme et donne dans le libéralisme au point d'effrayer Joseph Cauchon, le directeur du Journal de Québec, qui dut se départir de sa collaboration de peur de la réaction des lecteurs. Fréchette fonde alors deux journaux éphémères, le Drapeau et la Tribune 
de Lévis, où il s'inscrit dans la filiation idéologique des patriotes de 1837 1838. Il avait fait paraître en 1863 le deuxième recueil de poésie publié au Canada; mais Mes loisirs n'eut pas l'heur de plaire au public. Dégoûté littérairement et politiquement, le jeune homme décide au mois de juillet 1866 de quitter le Canada pour s'installer à Chicago comme journaliste et employé de bureau pour une compagnie de chemin de fer ${ }^{25}$. II y publie la Voix d'un exilé qui attaque férocement les Conservateurs alors au pouvoir au Canada et auteurs de la Confédération. Fréchette met fin à son exil volontaire en 1871 et brigue ensuite les suffrages sous l'étiquette libérale tant aux élections fédérales que provinciales. II devient député fédéral de Lévis en 1874. Après sa défaite de 1878, Fréchette décide d'abandonner la politique active pour se consacrer à la littérature. Un prix Montyon de l'Académie française couronne ses succès littéraires. Fréchette s'occupe également de journalisme et soutient des polémiques avec Chapman et l'abbé Baillargé. La survivance atténuée dè son nationalisme libéraliste et démocratique se manifeste encore timidement dans la Légende d'un peuple et l'incite à écrire une Petite Histoire des rois de France plus que tendancieuse. Il osera même chanter, lors du jubilé de la reine Victoria, les «vertus républicaines" de Sa Majesté (Marion, p. 37).

Bien que peu connu de nos jours, Adolphe-Basile Routhier fut pourtant un auteur et un magistrat célèbre au $\mathrm{XIX}^{\ominus}$ siècle. Également né en 1839, il étudie le droit en compagnie de Fréchette et voit rapidement s'ouvrir devant lui une brillante carrière dans la magistrature qui culminera avec le poste de juge en chef de la Cour supérieure de Québec et celui d'administrateur de la province en 1904-1905. II se mérite en outre le titre de "Sir". Les convictions religieuses conservatrices de Routhier le portent à rédiger en 1871 le Programme catholique de tendance ultramontaine radicale et à se présenter sans succès devant l'électorat de Kamouraska sous l'étiquette conservatrice. Critique littéraire, romancier apologiste, poète, essayiste religieux, auteur de récits de voyage et de théâtre historique, on lui doit de nombreux volumes, y compris un Nouveau Chemin de la croix ${ }^{26}$. A propos de cet auteur du texte français de l'hymne national canadien, Berthelot Brunet déclare: «Routhier n'aurait écrit que l'O Canada, ce serait péché véniel ${ }^{27}$." "Raseur dogmatisant et pompeux" (Baillargeon, p. 152), "prophète en retard du programme catholique» (Buies, p. 68), "homme du passé" (Dugas, p. 72), Routhier fait pourtant bonne figure dans les lettres de notre $X I X^{e}$ siecle. Si “ses écrits ont vite pris goût de rance" (Baillargeon, p. 152) et sont souvent superficiels, c'est parce qu'ils affichent cette sécurité que confèrent les systèmes de pensée totalisants, sécurité que le temps se charge généralement de ravir à ce genre d'écrits. Les cuvres de Routhier ont, sans doute à juste titre, été oubliées et ses idées n'auraient aujourd'hui aucune obédience; cependant, malgré ses petitesses, Routhier était sincère au point d'afficher, publiquement et peut-être à l'encontre de la jurisprudence, ses convictions ultramontaines lors de son célèbre jugement dans l'affaire des influences indues en période électorale. D'ailleurs, les théories qu'il soutenait ont trop dominé 
et empreint le monde intellectuel québécois pour que les écrits de «l'écrivain le plus prestigieux de l'école catholique canadienne» (Costisella, p. 189) soient simplement rejetés d'un revers de la main sous prétexte qu'ils ne sont plus acceptés d'emblée aujourd'hui.

II était donc inévitable que Fréchette et Routhier s'affrontent; les deux adversaires, véritables géants à la mesure du $\mathrm{XIX}^{\ominus}$ siècle québécois, représentent en effet deux grands courants de pensée qu'il faut situer dans le contexte intellectuel de l'époque afin de mieux en saisir les multiples ramifications.

Le Québec des années 1860 connaît une période d'effervescence généralisée. Littérairement, il vient d'assister à ce qu'il est convenu d'appeler sa naissance officielle dans les œuvres du groupe patriotique de Québec. Ce cénacle de tendance romantique rompt avec une tradition pseudoclassique qui ne recouvrait finalement qu'un manque à créer. Mais le romantisme au Québec fut d'abord politique. Dans le sillage de la Révolution française et de l'éveil des nationalités en Europe, les patriotes de 18371838 et leurs héritiers idéologiques, les Rouges, tentèrent, avec de moins en moins de succès, d'implanter au Québec des notions de républicanisme, de démocratie, d'anticléricalisme ainsi que de liberté individuelle et collective. Après les échecs politiques de 1837 et 1840, une littérature compensatoire animée d'un idéal nationaliste, parfois libéral et démocratique, voit le jour. Les forces conjuguées des radicaux ultramontains, des conservateurs de type tory britannique et des tenants d'un académisme littéraire improductif endiguent les idées novatrices tant du rougisme apparenté au libéralisme continental, que du libéralisme réformiste de type whig ou que du catholicisme libéral. Les forces libérales, conduites avec plus de panache que d'efficacité, iront de revers en échec, émousseront leur immanentisme radical au contact d'un transcendantisme omni-puissant et disparaîtront à toutes fins utiles de la scène québécoise.

En effet, les encycliques Quanta Curos et Mirari Vos ainsi que le Syllabus de Pie IX condamneront le catholicisme libéral. L'Institut canadien de Montréal, principal foyer de rougisme, sera pour sa part excommunié dès 1858 . Le projet conservateur de confédération recevra l'appui du clergé et entrera en vigueur én 1867. Le parti libéral rejettera de plus en plus le libéralisme européen et se définira finalement comme purement réformiste. Seul le romantisme littéraire subsistera, bien muselé par une censure religieuse, et ne s'exprimera, outre ses incidences stylistiques, que par une idéalisation du passé national et par l'exotisme véritable que fut la pseudo-canadianisation des lettres. Par la suite, un conservatisme modéré règnera au Québec jusqu'au milieu du $X X^{e}$ siècle et rappellera à l'occasion son transcendantisme extrémiste dans les écrits et gestes d'un Tardivel, d'un $M^{{ }^{g r}}$ Laflèche et, plus tardivement, d'un Groulx ou d'un Duplessis. Ce ne seront certainement pas les velléités immanentistes d'un Mercier; d'un Olivar Asselin, d'un Rodolphe Girard ou d'un Albert Laberge qui affaibliront cette emprise. D'ailleurs, s'il fallait une confirmation de la longue éclipse 
de l'immanentisme, il suffirait de constater le malaise des Roy, Dandurand, Klinck ou Marion (et ce jusqu'en 1958) face à la Voix d'un exilé pour se convaincre de la vitalité du transcendantisme contre lequel la Voix fit porter la dernière attaque immanentiste significative.

Le poème de Fréchette serait donc la voix d'un immanentisme exilé. Cependant, les implications profondes de ce court recueil de vers se dissimulent derrière un contenu somme toute assez facile à résumer. La Voix d'un exilé est une longue diatribe à l'adresse des Conservateurs francophones qui, artisans de la Confédération, auraient trahi leur peuple. L'auteur, que cette situation aurait forcé à s'exiler, chante également son amour et sa nostalgie du pays natal et invite son peuple à se régénérer et à rejeter les renégats qui l'entraînent à sa perte.

Dans la préface à l'édition de 1869, Fréchette avoue que "La Voix d'un Exilé n'est pas absolument originale [et que] c'est autant une imitation des Chátiments de Victor Hugo, qu'autre chose ${ }^{28}$ ». Pourtant la Voix n'est pas uniquement un démarquage canadien de l'œuvre de Hugo. Placée sous le patronage du géant du romantisme et nanti d'un titre qui évoque le thème résolument romantique de l'exil, l'œuvre témoigne évidemment d'une familiarité avec la production littéraire française immédiatement contemporaine. Mais, en s'inspirant spécifiquement des Chatiments, Fréchette adopte un parti pris de romantisme politique et d'opposition au pouvoir établi qui s'incarnera dans la réalité québécoise.

D'ailleurs Fréchette réitère ce romantisme politique en le canadianisant lorsqu'il dédie la première partie de son poème aux libéraux du Canada. Le poète précise sa pensée dans la dédicace de la deuxième partie: “Aux membres de l'Institut Canadien». Fréchette s'associe alors à la cellule rougiste par excellence, excommuniée par $M^{\text {gr }}$ Bourget, foyer d'anticléricalisme et instigatrice de l'affaire Guibord. Finalement, en offrant la troisième partie du poème à Louis-Joseph Papineau, il rejette l'ordre établi et s'inscrit dans le radicalisme extrémiste du chef patriote. Le texte de Papineau placé en exergue à la première partie du poème aurait déjà indiqué cette filiation idéologique, tout en rappelant que les causes de l'exil du poète résident dans un esclavage politique du peuple et appellent vengeance ${ }^{29}$. L'exergue à la deuxième partie, deux strophes des Chatiments, s'inscrit dans l'optique d'un romantisme vengeur et souligne la vilenie de l'adversaire conservateur ${ }^{30}$. Le troisième exergue, un extrait des Prophéties de Jérémie, s'il ne fait pas directement allusion au libéralisme religieux, se réclame d'une conception religieuse différente de celle du haut clergé approbateur du pacte confédératif ${ }^{31}$. En effet, la mission du prophète auquel s'identifie Fréchette consistait à venger les offenses faites au Seigneur, mais également à restaurer l'intégrité du peuple élu fourvoyé dans la voie du paganisme. Les trois sous-titres confirment ce que révélaient déjà les dédicaces et les exergues. "Adieux", "Consumatum est" et "Ultima verba" évoquent tour à tour un regret cuisant, un crime sacrilège comparable au meurtre du Christ et un romantisme politique 
hugolien ${ }^{32}$. Fréchette rejoint donc le romantisme révolutionnaire et se présente comme le vengeur appelé à renverser un ordre établi inique et corrompu. Selon le proverbe italien placé en page couverture, le poète se donne pour mission de secouer le mouton qu'est devenu le peuple avant que le loup conservateur ne le dévore ${ }^{33}$.

Après avoir si clairement annoncé ses couleurs, Fréchette, dans la première partie du recueil qui compte cent cinquante-six vers groupés en vingt-six sixains, plaint «[sa] patrie abandonnée aux mains d'une bande sordide". Selon le poète, les valeurs nationales ont été trahies et la religion profanée:

Mais ils ont souffleté nos gloires les plus pures;

Ils ont éclaboussé tous nos fronts immortels.

Aux croyances du peuple ils ont tendu des pièges,

Et dressé leurs tréteaux, histrions sacrilèges

Jusques à l'ombre des autels. (Voix, p. 9)

Fréchette en profite pour dénoncer la monarchie:

Le stigmate, il est vrai, décore bien la tête

Mais pas comme un bandeau royal. (Voix, p. 9)

Par contre, il glorifie «la sainte liberté " (Voix, p. 11) dont Papineau et les patriotes avaient pris la défence:

O toi qui survis seul à ces temps d'épopée

Que ta grande âme encore si fortement trempée

Doit souffrir en voyant cet âge d'apostats! (Voix, p. 10)

Le poète considère les États-Unis comme un modèle de république où brille une liberté absente au Canada. II déplore que l'avenir de son pays soit ainsi «au vent jeté " (Voix, p. 12) et termine ce premier chant en encourageant les libéraux canadiens. Comme dans chacune des autres parties du poème, la dernière strophe prédit le triomphe de la liberté:

Mais vous qui restez sur la brèche fumante,

N'allez pas, comme moi, céder à la tourmente,

Découragés, brisés, vaincus par les revers!

Leurs soldats sont nombreux: ne comptez pas les vôtres!

Songez que Jésus-Christ n'avait que douze apôtres,

Et qu'ils ont conquis l'univers! (Voix, p. 13)

Le but est noble et grand; le combat sera rude;

Mais bientôt, vous là-bas, moi dans ma solitude,

Nous verrons se lever le grand jour du réveil.

La voix des opprimés s'éleve grandissante...

Demain les nations, ô liberté puissante,

En pliant le genou, salueront ton soleil! (Voix, p. 14)

La deuxième partie, composée de deux cent vingt-deux vers groupés en trente-sept sixains, s'ouvre de nouveau sur une plainte mais qui exprime cette fois-ci la nostalgie que l'exilé éprouve à l'égard du pays qu'il a dû quitter: 
Toi qui fais les grands cœurs, au jour des grandes crises;

Toi qui chantes partout, sur les flots, dans les brises,

Toi qui fais pleurer l'exilé! (Voix, p. 18)

Le sort fait à son pays force le poète à oublier ces sentiments trop doux et à échanger la lyre pour le fouet. La longue suite d'injures que Fréchette adresse alors aux Conservateurs, plus particulièrement à ceux qui sont d'anciens patriotes, ne souffre aucun résumé:

A moi ce fouet sacré, ce fouet de la vengeance!

Arrière, scélérats! arrière, sale engeance!

Brigands de bas étage et fourbes de haut rang!

Point de grâce pour vous; fuiriez-vous jusqu'au pôle,

Je vous appliquerai le fer rouge à l'épaule,

Et je vous mordrai jusqu'au sang! (Voix, p. 20)

Le mal comme le bien a parfois grandi l'homme;

Le crime a ses héros... mais l'avenir les nomme Judas, Erostrate ou Mandrin!

Tout un peuple livré, là, sans pitié, sans honte,

Pour quelques écus, pour un titre de comte,

Pour quelque parchemin plus ridicule encor!...

Et, pour mettre le comble à ce scandale obscène,

Un triste aveuglement donne à l'horrible scène

Le sanctuaire pour décor! (Voix, p. 21)

Pour grossir dignement leurs cohortes impies,

Ils ont tout convoqué, requins, vautours, harpies,

Va-nu-pieds de l'honneur, bravos de guet-apens,

Hardis coquins, obscurs filous, puissants corsaires,

Bretteurs, coupe-jarrets, renégats et faussaires,

Ribauds, voyous et sacripants!

On voit, dans le repère où tout cela pullule,

Le ban, l'arrière-ban de toute la crapule;

Ils ont, pour les trouver, feuilleté les écrous,

Vidé les lupanars, sondé chaque tannière,

Bouleversé l'ordure, interrogé l'ornière,

Et plongé dans tous les égouts. (Voix, p. 21-22)

Dans une note placée à la fin du volume et qui renvoie aux vers précédents, Fréchette justifie la violence de ces vers en mentionnant des faits et des noms qui confirmeraient ses accusations. II réserve toutefois ses vers les plus acides à Sir Georges-Étienne Cartier:

Mais lui, le chef, qu'est-il, ce vantard hypocrite

Qui porte, sans rougir, tant d'infamie écrite

Sur son front impudent? Oui, qu'est-il, après tout?

Hargneux quand il se tait; insolent quand il parle;

Paillasse à Burlington, déserteur à Saint-Charles,

Rampant à Londres et gueux partout.

II a, pour parvenir, mis tout à son service;

Il escompte le vol, il pressure le vice,

Ce paillard tout suintant de prostitution;

Pour qu'il puisse à Windsor paraître en bas de soie,

Tout, le coffre public et la fille de joie

Sont mis à contribution. 
Déchirant par lambeaux nos libertés si chères,

II avait hardîment mis son peuple aux enchères, Et livré sa patrie à mille aventuriers:

Pour l'en récompenser, on le pare d'un titre

II se pâme, il se gourme, en son orgueil de pître:

Judas a ses trente deniers!

Iscariote ayant vendu son divin Maître,

Bourrelé de remords, il se pendit, le traître,

Croyant trouver au moins la paix dans le trépas;

Mais ce vil brocanteur n'a pas l'âme si tendre:

Jamais il n'aura, lui, le coeur d'aller se pendre...

II est plus lâche que Judas! (Voix, p. 23-24)

Dans la troisième partie qui comporte deux cent dix vers groupés en trente-cinq sixains, Fréchette se lamente toujours sur le sort pénible qui affecte sa patrie et rappelle les événements historiques qui en sont la cause. En contrepartie, il évoque le courage de son peuple:

Et, voyant ce troupeau qu'un escobar gourmande,

Le passant soucieux s'étonne et se demande

Si c'est bien là ce peuple aú passé glorieux,

Les fils de ces héros à la taille homérique,

Qui, sur tous les frontons de la jeune Amérique,

Gravaient leurs noms victorieux! (Voix, p. 34)

Cependant le peuple semble rester sourd à l'appel du poète et ne se révolte pas:

Et cependant tu dors, tu dors, et moi je pleure;

Triste, seul, loin des miens, j'attends que sonne l'heure

Où, piqué jusqu'au sang, tu te redresseras... (Voix, p. 37)

Mais avant de se taire et malgré sa déception, le poète réaffirme sa confiance inébranlable en la victoire de la liberté:

Ainsi j'allais rêvant, quand, céleste présage,

Comme une flèche d'or, déchirant le nuage,

Un rayon de soleil vint réchauffer mon front...

Que vois-je? I'arc-en-ciel! Est-ce la délivrance?

Qu'importe! c'est toujours un rayon d'espérance!

Ma lyre, attends encor, les beaux jours reviendront! (Voix, p. 38)

Voix toute lyrique que cette Voix d'un exilé où le poète étale sa colère, son amour et sa déception en des strophes de cinq alexandrins descriptifs ou explicatifs généralement suivis d'un octosyllabe qui frappe en médaillon la situation abhorrée ou adulée. Le poème prend ainsi une allure nerveuse où une majesté parfois pompeuse s'allie à une fougue à l'occasion trop oratoire. L'agressivité exacerbée alterne avec des moments de haute mélancolie où débordent une tendresse profonde et une dévotion sincère pour le sol national et son peuple. Les manques de goût occasionnels, les impropriétés de termes, une ponctuation souvent fluctuante, une métrique parfois hésitante, malheureusement caractéristiques des poètes de l'époque, déparent le texte; mais le contraste dans les sentiments et le ton situe ce poème à l'entrecroisement de la lyre et du fouet et lui confère une originalité exceptionnelle au $\mathrm{XIX}^{\mathrm{e}}$ siècle québécois. 
Certes, Hugo est plus que présent dans ces vers; l'auteur ne s'en est nullement caché. Daniel T. Skinner a consacré à ce sujet une thèse qui a le mérite d'être juste et de faire oublier l'odieux Lauréat de William Chapman ${ }^{34}$. Cependant cette dette marquée envers Hugo n'empêche pas le texte de participer au mouvement littéraire québécois des années soixante. Ne serait-ce que par son culte de la patrie et par l'évocation du passé historique, la Voix appartient pleinement à la littérature de notre $\mathrm{XIX}{ }^{\mathrm{e}}$ siècle. Mais l'on se doit également de remarquer que l'engagement politique et la violence du propos n'étaient pas sans précédent au Canada. Dans son excellent volume sur la poésie nationaliste, Jeanne d'Arc Lortie montre clairement que la poésie d'avant 1860 s'axait fréquemment sur la situation politique. Certains poèmes exhumés par ce critique s'apparentent nettement à la Voix. Un "pot-pourri » datant de 1810 en offre un exemple éloquent:

\section{Il est sorti d'une crasse profonde}

De noirs génies, des esprits de travers;

Pour nous tromper avec leurs langues immondes

Voudroient nous rendre ainsi qu'eux pervers ${ }^{35}$.

Michel Bibaud crut même nécessaire de consacrer son Épître l à l'abus de l'injure par les littérateurs ${ }^{36}$. Toutefois aucun autre texte avant la Voix n'avait atteint une telle ampleur dans la diatribe. Cet usage parfois outrancier de l'injure et le lyrisme exacerbé de l'auteur auraient déjà suffi à choquer des lecteurs encore imbus de classicisme. Mais les images vives, les comparaisons osées et les métaphores suivies ne reçurent pas meilleur accueil. Finalement le texte s'attaquait non seulement aux valeurs communément reçues mais prenait en outre le contre-pied du mode même de pensée officiel: les lecteurs et les critiques se rebiffèrent presque tous.

Les invectives dont sont l'objet les membres du parti conservateur outrèrent le public non pas tant comme une insulte partisane que comme une atteinte à travers eux à la Confédération et à l'ordre nouvellement et officiellement établi. Une telle attitude remettait en question la conception généralement acceptée du caractère sacré de l'ordre établi. Dieu étant la suprême autorité et l'ordonnateur de toute chose, l'autorité découle de lui. Cette autorité devient donc sacrée de par son origine et ses détenteurs ainsi que les institutions qu'ils ont créées, se sacralisent en participant de cette même origine divine. $M^{g r}$ Bourget exprime clairement cette conception à propos de la Confédération: «Aujourd'hui elle [l'Église] accepte sans réplique le gouvernement fédéral, parce qu'il émane de la même autorité [celle que Dieu impartit aux hommes]. " (Cité par Costisella, p. 135) Dans cette optique, la monarchie est la forme de gouvernement par excellence puisque le monarque représente la pérennité et l'incarnation directe de cette autorité au lieu d'en être simplement investi par la volonté populaire. Dans un tel système de pensée, l'autorité se fait transcendante, hiérarchique, incontestable et nie la souveraineté populaire.

Fréchette heurte de front cette conception transcendantiste car, non seulement il attaque les autorités établies, mais il accuse le clergé d'avoir 
trahi son rôle de protecteur du peuple en appuyant ces autorités. II condamne en outre la monarchie et vante les mérites du républicanisme américain démocratique et protestant. Pis encore, il s'inspire du Hugo des Châtiments, celui-là même qui vilipendait en Napoléon III le protecteur des États pontificaux qu'une poignée de zouaves québécois étaient allés défendre en 1868 et qui considérait de plus le pape Pie IX comme un abominable tyran.

On serait peut-être tenté de voir ici une simple confrontation entre une pensée laïque et une pensée religieuse. Certes le transcendantisme trouve son expression idéale dans une vision religieuse $\mathrm{du}$ monde; mais une pensée religieuse ne s'accompagne pas nécessairement d'une conception transcendantiste de l'autorité ${ }^{37}$. En fait, tant en 1860 que plus avant dans I'histoire du Québec, la pensée officielle se refuse à l'analyse de la situation concrète de la société québécoise et tente plutôt de trouver une justification du présent et de sa propre action en recourant à des principes intemporels qu'elle utilise (consciemment ou non) pour freiner l'évolution de cette société. Beaucoup plus qu'un simple conservatisme, cette attitude intellectuelle découle en partie d'une stagnation dans l'acquis qu'on sacralise en éternité et, en ce sens, le transcendantisme québécois correspond à l'état d'esprit d'une collectivité menacée.

L'appel immanentiste sape les fondements mêmes du transcendantisme en rejetant le caractère sacralisé de l'autorité et privilégie le présent plutôt que le passé. En somme, l'immanentisme récuse la sécurité que confère un système totalisant et propose plutôt des solutions qui ne garantissent pas un confort immédiat puisqu'elles n'ont pas encore subi l'épreuve du temps. Le poème de Fréchette témoigne donc de bien plus que d'une lutte partisane entre Libéraux et Conservateurs; car c'est en tant que pensée immanentiste que le rougisme de Fréchette s'oppose radicalement au conservatisme. Que l'adversaire le plus acharné du libéralisme ait été l'utramontanisme et non le parti conservateur confirme que l'opposition n'était pas partisane mais idéologique. L'ultramontanisme constitue en effet le transcendantisme le plus cohérent et le plus logique; à certaines occasions, il s'élève même contre le parti conservateur qui "dérogeait " à ses propres «traditions qui se résument dans un attachement inviolable aux doctrines catholiques et dans un dévouement absolu aux intérêts nationaux du BasCanada ${ }^{38}$ \%.

Que Routhier ait réagi violemment à la publication de la Voix n'a donc rien d'étonnant puisque Routhier se targue d'ultramontanisme. Ayant reçu de Fréchette la deuxième édition du poème, il s'empresse, sous prétexte d'amitié, de le critiquer dans le journal conservateur le Courrier du Canada ${ }^{39}$. Fréchette ayant pris connaissance de ces articles lui expédie la troisième édition avec cette remarque désobligeante: «Encore une occasion de faire de la réclame professionnelle et électorale." (Causeries, p. 233) Cependant, lorsque Routhier inclut ces articles critiques dans les Causeries du dimanche qu'il publie en 1871, Fréchette ne saurait plus se 
contenter de répondre par une simple remarque aussi acerbe soit-elle. Le poète est en effet rentré au Canada en 1871 et brigue les suffrages comme candidat libéral dans Lévis, alors que Routhier choisit pour sa part l'étiquette conservatrice dans Kamouraska. L'aspect immédiatement politique de la publication de Routhier exige une réplique et soulèvera une polémique qui déborde largement ce cadre électoral.

Si l'on s'en tient strictement à la critique littéraire de la poésie de Fréchette dans les Causeries du dimanche, ce livre ne justifierait pas à la rigueur une réplique de l'auteur de la Voix. Routhier loue d'abord le style de Mes loisirs bien qu'il considère ces poèmes comme de jolis vers sans profondeur. Quant à la Voix, il admire tant pour la forme que le message les vers dans lesquels Fréchette chante son pays. Mais son jugement est tout autre en ce qui concerne les vers vengeurs. $\mathrm{Ce}$ ne sont que vaines sonorités où Fréchette diffame le Canada et ses institutions. Selon Routhier, le poète encouragerait même l'assassinat politique parce que, dans le poème intitulé le Premier Coup de foudre, Fréchette voit le meurtre de D'Arcy McGee comme un avertissement populaire lancé aux auteurs de la Confédération. Si Fréchette avait joint les zouaves pontificaux et avait défendu les États papaux, quelle belle couvre eût été «la voix d'un zouave pontifical "; le poète a préféré vivre dans l'Amérique matérialiste et républicaine et n'a produit qu'une œuvre impie. Routhier exhorte le poète à ne pas céder à l'irréligion et au libéralisme et l'invite à revenir au pays. Mais la liberté que Fréchette prône dans la Voix, n'a pas de place au Canada: «La liberté démocratique n'a qu'un pied d'airain et c'est toujours sur des cadavres qu'elle le pose. C'est cette liberté que $M$. Fréchette veut introduire dans notre pays: merci poète, nous préférons la liberté religieuse posant ses pieds d'or sur le frontispice de nos temples." (Causeries, p. 229) Que le poète oublie ses erreurs de jeunesse et rentre dans le droit chemin et il fera une cuvre belle et bonne. Routhier s'attaque donc bien plus aux idées de Fréchette qu'à son œuvre.

Ces articles sur Fréchette figurent dans le cadre plus général des Causeries du dimanche où Routhier s'en prend violemment à la pensée libérale et affiche un ultramontanisme peut-être cohérent mais dont l'expression est par trop souvent puérile. Laurent a méchamment qualifié le livre de “plus beau dictionnaire de lieux-communs qui existe ${ }^{40}$ ". Buies s'en est également moqué. Sans résumer les Causeries qui aborde maints sujets allant du pouvoir temporel des papes à l'émigration canadienne-française en passant par Veuillot, Lamartine et Joseph Marmette, il faut toutefois en mentionner certains aspects qui aideront à comprendre la polémique ultérieure. Routhier y condamne Lamartine et Hugo pour leurs idées révolutionnaires ou immorales et va jusqu'à se demander «avec effroi quel peut être l'avenir d'une littérature qui a choisi Victor Hugo pour modèle". (Causeries, p. 212) Par contre, il exalte au plus haut point Louis Veuillot dont il se considère l'émule. Routhier démontre également que la défaite de la France en 1870 est due à la frivolité et à l'impiété des Français. II jette l'anathème sur toutes les idées modernes, particulièrement la démocratie, 
et considère que l'émigration canadienne-française vers les États-Unis est voulue de Dieu, ayant pour but de convertir au catholicisme cette république protestante, matérialiste et corrompue.

L'antithétisme des idées de Fréchette et de Routhier est on ne peut plus net. Systématiquement, ce qui pour l'un représente le mal, est chez l'autre un but louable; ce que le premier exalte, le second le vilipende. On pourrait établir une longue liste de ces oppositions : république / monarchie, libéralisme / conservatisme, démocratie / oligarchie, Hugo / Veuillot, modernisme / tradition, annexion ou indépendance / confédération, pouvoir de l'État / pouvoir de l'Église, libéralisme religieux / ultramontanisme, conjoncture historique / intervention divine, liberté / ordre établi, romantisme / mauvais goût, États pontificaux / Italie garibaldienne, France révolutionnaire / France de l'Ancien Régime, émigration malsaine / mission civilisatrice...

Deux systèmes de représentation du monde, deux idéologies, s'affrontent au point de transformer les mêmes réalités selon qu'on les inscrive dans un schème de pensée ou dans l'autre. Une allusion religieuse des deux auteurs en fournit une illustration cocasse. Fréchette avait comparé le petit nombre des libéraux à celui des apôtres du Christ en ces termes dans la Voix:

Songez que Jésus-Christ n'avait que douze apôtres

Et qu'ils ont conquis l'univers. (Voix, p. 13)

Routhier, quant à lui, dira dans le Centurion:

Il en est ainsi dans tous les mouvements révolutionnaires. C'est la minorité haineuse et violente qui terrorise la majorité, et qui gouverne. Au calvaire, elle était d'ailleurs commandée par des chefs puissants, les princes des prêtres, les scribes, les anciens $[. . .]^{41}$.

Les deux auteurs s'entendent sur le petit nombre des révolutionnaires; mais curieusement les révolutionnaires sont, selon Routhier, les autorités établies de l'époque et non le réformateur religieux qu'était alors Jésus. Selon l'optique de l'un ou l'autre adversaire. Jésus fut ou un révolutionnaire ou une victime des révolutionnaires. Même si Fréchette et Routhier étaient tous deux catholiques, leurs idéologies véhiculent chacune ses mythes propres et interprètent conséquemment de façon divergente le rôle d'un. même personnage.

C'est dans l'échange de lettres ouvertes qui suivit la parution des Causeries que s'exprime le plus clairement le heurt entre l'immanentisme de Fréchette et le transcendantisme de Routhier. Fréchette lança le premier les hostilités en intitulant ironiquement, comme le souligne Camille Roy, ses articles: Lettres à Basile. Tous saisirent l'allusion au personnage hypocrite et cupide du Barbier de Séville. Hugo avait utilisé le prénom en ce sens métaphorique dans les Chatiments; le malheur voulut pour Routhier qu'il s'appelât Adolphe-Basile et Fréchette eut la malice d'oublier le premier prénom pour ne s'en tenir, et abondamment, qu'au second, à la joie de ses 
partisans et au désespoir de Routhier qui y voit une "insultante familiarité». (Réponses, p. 62)

Cette première flèche de Fréchette sera suivie de bien d'autres et les deux auteurs feront mutuellement étalage d'esprit, de malice, de moquerie, de persiflage, d'argutie loufoque; ils relèveront leur mauvais style mutuel, souligneront leur ridicule réciproque, ne verront chez l'autre que manque d'intelligence, mauvaise foi ou fausseté et s'insulteront dans ces lettres qui ont fort bien vieilli. Toutefois, s'il faut admettre que Fréchette remporte la palme dans cet affrontement contre un Routhier qui se défend bien, ce dernier ne saurait être qualifié, comme l'a fait Dugas, «d'un peu sot, de cette sottise consacrée par les siècles et éternelle comme eux». (Dugas, p. 66) Si sottise il y a, c'est celle de toute une collectivité menacée qui tente de se définir à travers la pensée d'une élite elle-même dans une position précaire. La recherche de sécurité dans cette forme de pensée immuable qu'est le transcendantisme ne recouvre qu'un mécanisme de défense à la mesure d'un danger omniprésent de disparition.

Le ton presque continuellement moqueur de cet échange épistolaire ne doit pas nous leurrer; la polémique quitte rapidement le domaine littéraire et témoigne avant tout d'un conflit idéologique. N'ayant plus l'amitié pour excuse, Routhier durcit son jugement sur l'ensemble de l'œuvre de Fréchette. Ce dernier concède à Routhier que Mes loisirs "ça ne vaut rien". (Lettres, p. 53) Routhier juge que la Voix n'est qu'un «dévergondage de paroles» (Réponses, p. 75) et que Fréchette «sait son Victor Hugo sur le bout de ses doigts». (Réponses, p. 38) II suggère au poète de ne pas abuser de l'injure; Fréchette rétorque que Routhier ambitionne de jouer au Canada «le rôle que Veuillot s'est arrogé en Europe» (Lettres, p. 45) et que ce maître ne dédaigne pas l'usage d'insultes beaucoup plus corsées que celles dont il s'est lui-même servi dans la Voix. La question stylistique importe finalement assez peu puisque Routhier veut surtout démontrer qu' « un honnête hoimme devrait rougir d'[...] avoir écrit » la Voix. (Réponses, p. 73) II semble que Fréchette lui ait donné raison par son attitude ultérieure face à son cuvre. Déjà il avoue que la Voix «fait peut-être mauvais genre» et que «la situation canadienne n'est plus la même qu'à sa parution ». (Lettres, p. 101, 139)

La susceptibilité littéraire de Fréchette a certainement été blessée par les critiques de Routhier. Quand Routhier insinue que Fréchette a voulu lui ravir sa place dans le monde littéraire québécois, Fréchette le récuse en invoquant le manque de talent de Routhier qui n'a su se mériter qu'un troisième prix à un concours littéraire organisé par l'Université Laval; il y a une note de dépit dans cette réplique car Fréchette n'avait encore connu aucune reconnaissance littéraire officielle à l'époque. La querelle glisse alors sur un terrain beaucoup plus personnel où les accusations et insinuations de tout ordre foisonnent. Routhier accuse Fréchette de lâcheté pour avoir abandonné le Canada et voit une preuve de la faiblesse de son adversaire lorsque Dessaules intervient dans leur débat. Fréchette, pour sa part, laisse planer plusieurs doutes sur la vertu et l'honnêteté de Routhier, 
surtout pendant les campagnes électorales. II accuse même formellement Routhier d'avoir écrit une lettre anonyme au curé Patry de Saint-Pascal et de tenter de contourner l'autorité de l'évêque de Québec.

L'injure n'effraie pas les deux adversaires qui en inondent copieusement leur rival: «fou, arriviste, envieux, calomniateur, mal éduqué, ignorant, plagiaire, sans honneur, Don Quichotte, lièvre, fat, race de vipère, lâche insulteur, énergumène, méduse, voleur d'élection, pharisien, vendeur du temple, cafard intriguant " et autres aménités sont allègrement échangées. Une telle accumulation ne laisse cependant pas soupçonner la drôlerie et parfois la finesse du trait. II est d'un charretier de qualifier son rival de rat; Fréchette adopte une savante drôlerie:

Qui sait, peut-être pourra-t-on un jour dire de vous [Routhier] ce que le bon Lafontaine disait d'un rat célèbre:

C'était un vieux routier: il savait plus d'un tour

Même s'il avait perdu sa queue à la bataille.

II manque une $h$ mais la scie y est. (Lettres, p. 143)

Le chef-d'œuvre du genre revient toutefois à Routhier. Après certains raisonnements prouvant que Fréchette a “avalé ses accusations", Routhier termine par cette apostrophe: «Donc, M. Fréchette, avalé le pot aux roses ". (Réponses, p. 98) Or le contenu de ce pot aux roses avait servi à. «badigeonner des peintures rien moins qu'odoriférantes n à la porte de Routhier. Voilà qui est fort élégamment dit; un premier ministre du Canada n'eût pas fait mieux. Somme toute, rien de bien sérieux dans ces empoignades d'ailleurs fréquentes à l'époque. La malice d'écrits comme Portraits et pastels littéraires, Profils et grimaces ou Silhouettes littéraires témoigne de la bonne fortune du genre; Augustin Laperrière a même pu reproduire deux volumes de Guêpes canadiennes.

Les incidences personnelles ajoutent du piquant au conflit mais ne le justifient pas. Ces attaques tiennent au genre polémique adopté et l'essentiel du conflit réside dans le choc des idées. Comme l'affirme Fréchette, chaque auteur s' «occupe non pas précisément de [l'adversaire] mais de ce qu'[il] dit». (Lettres, p. 19) Les protagonistes se considèrent comme les porte-parole de partis ou d'écoles adverses et attaquent ces groupes à travers leur représentant. La polémique se fait alors partisane au sens étroit du mot et remonte fort loin puisque, selon Routhier, «Adam [...] était conservateur avant sa chute et libéral après". (Lettres, p. 25) Le fait que Buies, rouge flamboyant, se soit également moqué de Routhier et que Dessaules succède à Fréchette dans ses échanges avec Routhier, confirme cette partisanerie. D'ailleurs Fréchette avait déjà accusé Routhier de le critiquer à des fins électorales et déclare lui répondre «au nom de tout un parti politique». (Lettres, p. 41) Routhier reconnaît en son adversaire "le chef du parti libéral [qui] déclare la guerre à toute l'école catholique». (Reponses, p. 57) Routhier, prête-main des Conservateurs "qui le soudoient" (Lettres, p. 43), utiliserait la religion à leur profit: «J'ai dit qu'il fallait combattre les libéraux et que le libéralisme est une hérésie. Je le 
dis encore après Pie IX, et je ne le rengaine pas." (Réponses, p. 96) Évidemment la distinction entre libéral et oppositionniste n'échappe pas à Routhier; mais Fréchette précise que, pour être oppositionniste, il suffit de renier les libéraux et d'embrasser le parti conservateur. Si Routhier attaque le parti libéral "au nom de la religion", c'est "parce que [ce parti] est opposé au ministère actuel». (Lettres, p. 129) Le poète affirme même: «Tout commissaire de Satan que je sois, si je passais au ministère aujourd'hui pour demain, tous les Basiles du pays seraient les premiers à me décerner les plus beaux certificats d'orthodoxie imaginables. " (Lettres, p. 129-130)

La composante partisane est donc des plus importantes dans ce conflit; mais de l'aveu même des pugilistes, leur querelle ne saurait se limiter aux cadres d'une politique étroite. Si le nom du parti libéral contribue à maintenir une certaine ambiguïté face à la religion, ni l'un ni l'autre polémiste n'en est dupe. Outre sa distinction entre libéral et oppositionniste, Routhier reconnaît que son adversaire véritable n'est pas le parti libéral mais bien «toute la petite presse voltairienne, sceptique et libérale [qui] est liguée contre [Routhier]». (Réponses, p. 82) Quant à Fréchette, il précise que sa "satire [est] beaucoup plus dirigée contre [une] école hypocrite envieuse et servile que contre le parti purement politique qu'on appelle conservateur ". (Lettres, p. 104) D'ailleurs la Confédération, œuvre des Conservateurs et occasion première du conflit, est presque totalement passée sous silence dans le débat.

Si donc l'aspect littéraire ne sert que de prétexte, si les attaques personnelles ne sont pas de prime importance et si la composante partisane ne justifie pas des oppositions si radicales, il faut croire, comme le laissent déjà soupçonner les textes cités, que cette lutte illustrerait des conceptions philosophico-religieuses opposées. Dans sa première lettre, Fréchette s'était moqué des conceptions simplistes de la Providence que formulait Routhier. Là où Roùthier voit le doigt de Dieu, Fréchette décèle un concours de circonstances plus ou moins fortuites. Le heurt entre l'immanentisme et le transcendantisme se manifeste ici de façon schématique.

Ces deux conceptions ont des conséquences politiques directes qui ne se ramènent pas à la simple collusion du parti conservateur et des ultramontains. Fréchette ne voit que mauvaise foi dans cette alliance lorsqu'il applique à Routhier ces paroles de Balmes: «Je suis profondément convaincu qu'on met en danger les intérêts du catholicisme, toutes les fois qu'on l'identifie, en faisant son apologie, avec une cause politique quelconque." (Lettres, p. 54) Le poète se fourvoie alors dans sa partisanerie; car c'est l'essence même du parti conservateur et de l'ultramontanisme qui les oppose au parti libéral de l'époque. De par leur transcendantisme, tous deux sacralisent l'univers: "On sait que partout où le libéralisme n'a pas encore pénétré, le roi et le prêtre sont deux personnes inviolables et sacrées. " (Réponses, p. 76) Dans l'optique de Routhier, toute dérogation au principe d'autorité fondé sur le transcendantisme est condamnable: 
Liberté, Égalité, Fraternité, telle est la semence jetée par Washington qui fera du Nouveau Monde le vrai berceau du genre humain. Jusqu'à présent, nous avions cru que le vrai berceau du genre humain était l'orient et que c'était Jésus qui y avait jeté la semence féconde. Nous étions dans l'erreur. La monarchie! la noblesse! vieilleries que tout cela! (Réponses, p. 77)

Qu'il s'agisse de toutes les formes de libéralisme ou du texte de la Voix, le transcendantisme les recouvre d'une même réprobation et Routhier, conservateur ultramontain, donne de plain-pied dans le transcendantisme. Certes, Routhier ne saurait soutenir, comme le veut Fréchette, qu'il soit "impossible d'être catholique sans être monarchiste, sans soutenir les privilèges de castes" (Réponses, p. 114); mais il réplique que, si la république peut exister, elle doit être catholique. Cette république sera soumise aux autorités ecclésiastiques, émanation directe de Dieu. On comprend alors que la Confédération ne puisse être une "œuvre immonde applaudie par Satan" car c'est «un changement politique que nos évêques approuvaient». (Réponses, p. 144) La responsabilité gouvernementale de nature démocratique entre difficilement dans de tels cadres et l'opposition n'a pas droit de cité. En somme, la société humaine doit être tributaire de la société religieuse parce que «la séparation de l'Église et de l'état est une doctrine absurde et impie». (Programme catholique) II est alors normal que l'autoritarisme règne et s'oppose à toute velléité de changement; en effet, le changement, s'il n'est en soi mauvais, repose sur des justifications qui rejettent un ordre sacralisé et, à travers cet ordre, porte atteinte à l'Origine même de la sacralisation. Les autorités cléricales bénificient au premier chef d'un tel système et l'intervention généralisée des clercs dans toutes les sphères d'activité n'est que la concrétisation de ce transcendantisme. Les accusations d'irréligion portées contre Fréchette et les libéraux, d'une portée catastrophique durant cette période de catholicisme intense, recouvrent finalement un interventionnisme clérical fondé sur le transcendantisme et non une défense de la religion elle-même.

La problématique religieuse ne doit pas égarer le lecteur; ce conflit ne doit pas être assimilé à une opposition entre la religion et le laïcisme. Fréchette, tout comme Routhier, est catholique. La pléthore de théologiens cités de part et d'autre ainsi que le recours aux Pères de l'Église suffiraient à confirmer que leur débat s'inscrit dans l'univers intellectuel de l'époque qui était foncièrement religieux. Mais, à l'intérieur même de ce cadre religieux, deux types de pensée s'affrontent.

Fréchette et Routhier ne s'opposent pas tant au sujet des principes d'action qu'au sujet du mode de justification de ces principes. Le transcendantiste Routhier croit que ces principes découlent d'une autorité intemporelle, fixiste et hiérarchisée, alors que l'immanentiste Fréchette cherche à adapter ces mêmes principes à une évolution historique. Les incidences multiples et concrètes de ces deux démarches s'incarnent dans deux visions de la société québécoise de l'époque. Il est symptômatique que Mason Wade ${ }^{42}$ ait vu dans la Voix un manifeste annexionniste alors qu'il 
s'agit d'un plaidoyer anti-confédératif qui prône un républicanisme québécois. Fréchette y vante les vertus des États-Unis pour montrer que la démocratie est valable et productive et pourrait s'appliquer au Québec. (Comme les Rouges, Fréchette a évidemment été tenté par l'annexionnisme ${ }^{43}$.) Le poète exalte les patriotes et les révolutions française et américaine parce qu'il reconnaît aux peuples le droit de se diriger eux-mêmes. II condamne l'alliance du clergé et des Conservateurs parce qu'il refuse l'ingérence cléricale indue dans la vie politique et historique de la nation. En somme, Fréchette s'oppose à toute autorité qui ne tire sa vertu que d'un canon immuable.

Au-delà des manifestations littéraires, personnelles, partisanes ou philosophico-religieuses, ce sont deux mécanismes de pensée qui s'affrontent en Fréchette et Routhier. Que Fréchette ait par la suite adouci ses positions, la chose est déjà perceptible dans les Lettres à Basile, et se soit ultérieurement réfugié dans le nationalisme passéiste de la Légende d'un peuple, indique la victoire, chez l'auteur même de la Voix, du transcendantisme que soutenait Routhier. Car qu'il s'agisse de sacralisation d'origine religieuse, traditionaliste ou nationaliste passéiste, le transcendantisme brille en inaltérables credos de pensée au Québec. Comme l'a écrit Joseph Costisella en accordant trop de poids à l'Église dans cette situation: «Désormais et jusqu'à la fin du dix-neuvième siècle sinon même en plein vingtième siècle, existeront, de par l'autorité du haut clergé, les péchés mortels de Liberté, Démocratie, Égalité, Souveraineté du peuple, etc. " (Costisella, p. 43)

La Voix d'un exilé et la polémique qui s'ensuivit constituent la dernière manifestation immanentiste d'importance car, expression du romantisme politique, l'immanentisme disparaîtra de la scène québécoise avec le rougisme et l'Institut canadien pour ne laisser subsister qu'un parti libéral “oppositionniste». Laurier, dans son célèbre discours du 26 juin 1877 intitulé le Libéralisme politique, consacrera la disparition de l'immanentisme:

Je sais que, pour une partie de nos compatriotes, le parti libéral est un parti composé d'hommes à intentions droites peut-être, mais victimes et dupes de principes par lesquels ils sont conduits inconsciemment, mais fatalement à la révolution. [...] L'esprit humain sera attiré dans des directions opposées, par le charme de l'habitude ou par le charme de la nouveauté [...]: les premiers sont les conservateurs; les seconds sont les libéraux. [...] D'un côté vous avez ceux qui gouvernent et de l'autre ceux qui surveillent ${ }^{44}$.

L'Événement, le journal même qui publia les Lettres à Basile, se réjouit de cette nouvelle orientation:

Nous savons où nous allons désormais; nous n'allons pas aux cataclysmes révolutionnaires; le libéralisme est dégagé de ses aspects farouches, de son caractère anti-social et anti-religieux, et il ne garde plus que sa physionomie véritable, celle de l'amour des libertés légi- 
times et nécessaires, des libertés progressives, qui résultent des conditions naturelles du progrès, et non des brusques poussées en avant que veulent imprimer des esprits dangereux. (Willison, p. 399)

Le parti libéral renie donc ses racines immanentistes radicales pour envisager un partage de juridiction avec l'Église: la politique ne souffrira plus des interventions cléricales et les Libéraux ne s'occuperont que de politique au sens strict. Ce compromis typique de Laurier, qui oubliait sa jeunesse rougiste et anti-confédérative, ne sera pas accepté d'emblée en 1877 par la partie adverse et principalement par son aile ultramontaine, bien que le libéral MacKenzie ait détenu le pouvoir à Ottawa de 1874 à 1878 à la suite du scandale des chemins de fer. Malgré cette concession libérale et si l'on excepte les deux éphémères cabinets provinciaux de Joly de Lotbinière de mars 1877 à octobre 1878, le parti libéral tant au provincial qu'au fédéral devra attendre jusqu'en 1896 pour conquérir le pouvoir. Laurier, élu en dépit de l'épiscopat catholique qui condamnait la position du chef libéral en ce qui a trait au problème des écoles du Manitoba, en appellera à Rome de l'ingérence politique des évêques. L'année suivante, Mgr Merry del Val, délégué du Vatican, consacrera le partage de juridiction de 1877 et imposera silence aux radicaux ultramontains. Le transcendantisme perdait peu dans ce marché. En effet, la constitution canadienne, par la séparation des pouvoirs entre les provinces et l'État fédéral, avait déjà placé sous la juridiction provinciale les sphères d'action les plus importantes aux yeux du clergé et le transcendantisme pourra de toute façon régner en maître dans la province de Québec, à l'abri de toute intervention extérieure.

Le poème de Fréchette témoigne donc de la violente réaction d'impuissance d'un immanentisme qui se sait déjà condamné et qui devra attendre Refus global pour retrouver sa virulence d'antan:

Un petit peuple serré de près aux soutanes restées les seules dépositaires de la foi, du savoir, de la vérité et de la richesse nationale. [...] Le passé dut être accepté avec la naissance, il ne saurait être sacré. [...] ... chaque fois qu'un homme consent à être un homme neuf dans un temps nouveau. Définition de tout homme, de tout temps ${ }^{45}$.

Les conséquences littéraires de cette domination transcendantiste se reconnaissent facilement dans la censure morale qui pesait sur les œuvres jusqu'à très récemment. Mais le manque d'emprise sur la réalité concrète dont faisait preuve la littérature québécoise relèvé du même phénomène. L'irréalisme foncier des romans de la terre qui cherchaient à dépeindre et à implanter une société idéalisée et sans rapport avec la vie réelle témoigne d'une démarche transcendantiste. Des valeurs immuables ont été définies par autorité et si la réalité les contredit, la réalité a tort. Tout le régionalisme canadien souffre de ce même mal et devient un exotisme au même titre qu'un poème du Paon d'émail. La tragique destinée d'un Saint-Denys Garneau n'est pas non plus étrangère à ce curieux refus du concret que fut le transcendantisme. Que le transcendantisme extrémiste se soit allié à un nationalisme conservateur pour donner naissance à un roman d'anticipation comme Pour la patrie témoigne d'un irréalisme et d'un interventionnisme transcendantiste intégral. 
Dans un tel contexte, le destin de la Voix d'un exilé n'est plus mystérieux. L'œuvre fut reniée, oubliée, mésestimée, parce qu'elle remettait en question la sécurité intellectuelle de toute une collectivité et menaçait un ordre social péniblement constitué sous la pression d'une réalité menaçante. Marcel Dugas a cru voir. dans la polémique entre Fréchette et Routhier une transposition de querelles européennes: "Ces deux combattants fougueux qui s'injurient sont émouvants à leur manière, car ils reproduisent outre-atlantique, les grandes batailles d'idées qui se livrent en France." (Dugas, p. 78) S'il est possible, toutes proportions gardées, de voir en Fréchette un Hugo local et en Routhier un Veuillot canadien, il faut cependant admettre que la signification et les conséquences de leur débat prennent place dans une problématique québécoise qui continue, peut-être à notre insu, à nous hanter.

Guy Monette

R.M.C., Kingston

1. Nous utilisons l'adjectif transcendantiste et le substantif transcendantisme pour désigner des idéologies, des théories ou des mécanismes de pensée dont les fondements clairement énoncés ou manifestement explicites reposent sur des valeurs immuables grâce auxquelles ces idéologies, théories ou mécanismes interprètent la réalité, orientent leur activité et proposent des solutions aux diverses situations auxquelles ils sont confrontés. Ce néologisme à la fois précis et descriptif mais facilement compréhensible et suffisamment extensif nous est apparu nécessaire pour regrouper les aspects tant littéraires, politiques, religieux, sociaux ou philosophiques des divers courants de pensée qui ont dominé le Québec de la deuxième moitié du $\mathrm{XIX}^{\ominus}$ siècle à la deuxième moitié du XXe. Par opposition, des idéologies, théories ou mécanismes de pensée qui se fondent sur une analyse des faits et s'élèvent contre la domination des arguments d'autorité au nom de la réalité seront dits immanentistes; le substantif correspondant est immanentisme.

2. Henri d'Arles, Makers of Canadian Literature: Louis Fréchette, Toronto, Ryerson Press, s.d., p. 12. Dorénavant dans notre texte: Arles.

3 Auguste Viatte, Histoire littéraire de l'Amérique française des origines a 1950, Paris, PUF, 1954, p. 126.

4. Voir Gérard Tougas, Histoire de la littérature canadienne-française, Paris, PUF, 1967, p. 41; Samuel Baillargeon, Litterature canadienne-française, Montréal, Fides, 1957, p. 106-108. Dorénavant dans notre texte: Baillargeon; Charles ab der Halden, Etudes de littérature canadienne-française, Paris, Rudeval, 1907, p. 230; Albert Dandurand, la Poésie canadienne-française, Montréal, Albert Lévesque, 1933, p. 71 et 77 ; Pierre de Grandpré et al., Histoire de la littérature française du Québec, Montréal, Beauchemin, 1971, p. 206; Paul Wyczynski, "Dans les coulisses du théâtre de Fréchette", in Archives des lettres canadiennes I, Ottawa, PUO, 1961, p. 105.

5. Marcel Dugas, Un romantigue canadien, Louis Fréchette (1839-1908), Montréal, Beauchemin, 1946, p. 41. Dorénavant dans notre texte: Dugas.

6. Narcisse-Eutrope Dionne, Inventaire chronologique des livres et journaux, etc., publiés en langue française dans la Province de Québec 1764-1906, Québec, s.é., 1905, p. 63. lan Forbes Fraser, Bibliography of French Canadian Poetry, Part 1: From the Beginnings of the Literature through the Ecole litteraire de Montréal, New York, Columbia University, 1935, p. 64.

7. Adolphe-Basile Routhier, les Causeries du dimanche, Montréal, Beauchemin et Valois, 1871, p. 233. Dorénavant dans notre texte: Causeries. 
8. Ces différentes éditions offrent des variantes dont certaines découlent des remarques stylistiques que Routhier fit à l'occasion des deuxième et troisième éditions.

9. Les journaux libéraux reproduisent avec joie la Voix d'un Exilé. La plus ancienne reproduction se trouve dans le Pays du 27 mars 1867, vol. 16, $n^{\circ} 31$. Comme l'édition de 1866 est devenue introuvable, il faut considérer cette reproduction comme le plus ancien état du texte.

10. «Dans Le Monde, journal de Paris, P. de Cazes reproduisit la plupart de ces strophes véhémentes et les fit suivre de considérations élogieuses sur le lyrisme du poète canadien. " Séraphin Marion, les Lettres canadiennes d'autrefois, tome IX, Ottawa, Éclair et Université d'Ottawa, 1958, p. 26. Dorénavant dans notre texte: Marion.

11. Henri d'Arles, Essais et conférences, chez l'auteur, 1909, p. 98.

12. "J'ai le regret d'aninoncer à mes souscripteurs que l'auteur, M. Fréchette, m'ayant exprimé le désir de ne pas donner suite, du moins pour le présent, à ma promesse de publier dans ce volume cette "guêpe politique", $j$ 'ai dû́ céder à son désir par simple délicatesse." Augustin Laperrière, les Guêpes canadiennes, tome 2, Ottawa, A. Bureau, 1882.

13. Edmond Lareau, Histoire de la littérature canadienne, Montréal, John Lowell, 1874, p. 120,124 . Dorénavant dans notre texte: Lareau.

14. William Chapman, le Lauréat, Québec, Léger Brousseau, 1894, p. 34, 65.

15. Camille Roy, Poetes de chez nous, Montréal, Beauchemin, 1934, p. 50, 25, 16 , 61,24 . Dorenavant dans notre texte: Roy.

16. «Nous avons eu le bonheur de retrouver la version originale de La Voix d'un Exilé que nous n'avons vue citée nulle part. Là surtout les variantes étaient révélatrices. m Michel Dassonville, Fréchette, Montréal, Fides, 1959, p. 14.

17. Gérard Bessette, L. Geslin et Ch. Parent, Histoire de la littérature canadiennefrançaise par les textes, Montréal, CEC, 1968, p. 120. Voir pour plus de détails, Gérard Bessette, les Images en poésie canadienne-française, Montréal, Beauchemin, 1972, p. 108-109.

18. Georges A. Klinck, Louis Fréchette prosateur, Lévis, Le Quotidien, 1955, p. 65. Dorénavant dans notre texte: Klinck.

19. Joseph Costisella, l'Esprit révolutionnaire dans la littérature canadienne-française de 1837 à la fin du xıxe siecle, Montréal, Beauchemin, 1968. Dorénavant dans notre texte: Costisella.

20. Louis-Honoré Fréchette, Lettres \& Basile, Québec, L'Événement, 1872. Ces lettres sont également reproduites dans les Guêpes canadiennes, tome 2, pages 19 à 162. Nous citons cette reproduction. Dorénavant dans notre texte: Lettres.

21. Ces lettres sont reproduites dans les Guêpes canadiennes avec les Lettres à Basile. Dorénavant dans notre texte: Réponses.

22. Routhier ayant attaqué Louis-Antoine Dessaules dans une des lettres qu'il adressait à Fréchette, Dessaules écrivit deux lettres à Routhier pour rétablir les faits. Ces deux lettres sont également reproduites dans les Guêpes canadiennes avec celles de Fréchette et Routhier. Dessaules était le chef des Rouges et l'animateur de l'Institut canadien de Montréal.

23. Arthur Buies, Chroniques, humeurs et caprices, Québec, C. Darveau, 1873, p. $68,91,101$. Dorénavant dans notre texte: Buies.

24. Jean Piquefort, "Louis Fréchette" in Portraits et pastels littéraires. Voir note 39.

25. Ce départ généralement interprété comme une manifestation de dépit pourrait avoir une autre explication. S'appuyant sur l'Histoire de la presse franco-américaine d'Alexandre Bélisle, Marcel Dugas écrit que Fréchette aurait, sans le savoir, servi de guide à un espion ténian à Québec. Apprenant par la suite la véritable identité de son hôte, le poète aurait pris peur et se serait enfui aux États-Unis. Nous n'avons pu retracer l'origine de cette allégation même si Dugas affirme que Fréchette a expliqué lui-même les raisons de sa fuite. (Dugas, p. 33)

26. Les Échos; les Causeries du dimanche; Portraits et pastels littéraires; $\dot{A}$ travers I'Europe; À travers l'Espagne; Conférences et discours; De Québec à Victoria; Paulina; le Centurion; De l'homme a Dieu; Montcalm et Lévis, etc.

27. Berthelot Brunet. Histoire de la littérature canadienne-française, Montréal, HMH, 1970 , p. $52-53$. 
28. Louis-Honoré Fréchette, la Voix d'un exilé, Chicago, L'Amérique, 1869. Nous citons abondamment ce texte parce qu'il n'est pas facilement disponible. Dorénavant dans notre texte: Voix.

29. «Ceux qui, aujourd'hui, s'exilent en si grand nombre, parce que le dégoût pour les hommes et les mesures actuels les poussent à aller respirer un air plus pur, disent quels sont les stigmates que le colon porte au front... lis donneront de plus en plus des consolations et des espérances aux opprimés: ils avancent I'heure des rétributions, l'heure des nobles vengeances, où le bien sera fait même à ceux qui ont pratiqué le mal." Nous ne savons pas de quel discours de Papineau est extrait cet exergue. (Voix, p. 7)

30. "L'orgie a rougi leur moustache; La bombance après l'équipée!

Des rouleaux d'or gonflent leur sac; On s'attable; hier on tua...

Pour capitaine ils ont ganache;

Ils ont Cocagne pour bivouac.

¿ Napoléon, ton épée $s^{\prime}$ agit des deuxième et troisième strophes de la quatrième partie du poème intitulé "À l'obéissance passive" qui figure dans les Châtiments de Hugo. (Voix, p. 17)

31. « Usquoque lugebit terra, et herba omnis regionis siccabitur, propter malitiam habitantium in ea?" (Voix, p. 29) La Bible de Jérusalem traduit par: "Jusques à quand la terre sera-t-elle en deuil, et l'herbe de la campagne séchera-t-elle? Á cause de la méchanceté des gens qui y habitent... " Jérémie XII, 14.

32. "Ultima verba" est le titre du dix-septième poème du septième livre des Chatiments.

33. "Che si pecora fa, il lupo la mangia", ce que nous traduisons littéralement par «Celui qui se fait mouton, le loup le mange».

34. Daniel T. Skinner, "The Poetic Influence of Victor Hugo on Louis Fréchette", thèse inédite de Ph.D., Harvard University, 1952.

35. Jeanne d'Arc Lortie, la Poésie nationaliste au Canada trançais (1606-1867), Québec, PUL, 1975, p. 146. On consultera aussi p. 209, 226, 227, 278, 280, 302 .

36. Michel Bibaud, Épitres, satires, chansons, épigrammes et autres pieces de vers, Montréal, Réédition-Québec, 1969, p. 56-66. L'original date de 1830.

37. Les théologies de la libération actuellement en vogue en Amérique latine ne peuvent être qualifiées de transcendantistes dans leur démarche même si elles admettent Dieu comme principe suprême.

38. Adolphe-Basile Routhier, "le Programme catholique", l'Événement, 20 avril 1871. Dorénavant dans notre texte: Programme catholique.

39. Routhier, sous le pseudonyme de Jean Piquefort, réaménagera ses critiques sur Fréchette dans Portraits et pastels littéraires publié en 1873 dans le Courrier du Canada. Ce portrait de Fréchette ainsi que ceux d'autres écrivains sera republié chez Léger Brousseau à Québec dans la troisième livraison des Portraits et paste/s littéraires. Le texte est également repris dans Augustin Laperrière, les Guêpes canadiennes, tome I, Ottawa, A. Bureau, 1881, p. 320-332.

40. Laurent (pseudonyme de François-Alexis-Hubert Larue et Henri-Raymond Casgrain), Profils et grimaces in les Guêpes canadiennes, tome 2, p. 11.

41. Ádolphe-Basile Routhier, /e Centurion, Paris, Société Saint-Augustin et Desclée de Brouwer, 1909, p. 405.

42. Mason Wade, les Canadiens français de 1760 à nos jours Montréal, CLF, 1966, p. 423.

43. Robert Rumilly, Histoire de la procince de Québec, tome 1, Montréal, Bernard Valiquette, 1940 , p. 146.

44. J.S. Willison, Sir Wilfrid Laurier and the Liberal Party, Toronto, George N. Morang, 1903, vol. 2, p. 401-438. Le texte est reproduit en français.

45. Paul-Émile Borduas, Refus global, Shawinigan, Anatole Brochu, 1972, p...11, 20,21 . La première édition date de 1948. 